\title{
Meta
}

Journal des traducteurs

Translators' Journal

\section{L'enseignement de la théorie de la traduction : quelques réflexions pédagogiques}

\section{Agnès Whitfield}

Volume 48, numéro 3, septembre 2003

Traduction et enseignement

Translation and teaching

URI : https://id.erudit.org/iderudit/007603ar

DOI : https://doi.org/10.7202/007603ar

Aller au sommaire du numéro

Éditeur(s)

Les Presses de l'Université de Montréal

ISSN

0026-0452 (imprimé)

1492-1421 (numérique)

Découvrir la revue

Citer cet article

Whitfield, A. (2003). L'enseignement de la théorie de la traduction : quelques réflexions pédagogiques. Meta, 48(3), 429-437. https://doi.org/10.7202/007603ar
Résumé de l'article

Cet article examine, à partir d'une expérience pédagogique réalisée dans le contexte canadien, le rôle d'un cours de théorie au sein d'un programme de formation professionnelle. Le cours proposé est conçu en fonction des besoins des apprenants. Une approche sociale et engagée de la théorie remplace la perspective textuelle traditionnelle. Le contenu didactique du cours ainsi que la démarche pédagogique sont analysés et évalués. 


\title{
L'enseignement de la théorie de la traduction: quelques réflexions pédagogiques
}

\author{
AGNÈS WHITFIELD \\ Université York, Toronto, Canada \\ agnes.whitfield@videotron.ca
}

\begin{abstract}
RÉSUMÉ
Cet article examine, à partir d'une expérience pédagogique réalisée dans le contexte canadien, le rôle d'un cours de théorie au sein d'un programme de formation professionnelle. Le cours proposé est conçu en fonction des besoins des apprenants. Une approche sociale et engagée de la théorie remplace la perspective textuelle traditionnelle. Le contenu didactique du cours ainsi que la démarche pédagogique sont analysés et évalués.
\end{abstract}

\section{ABSTRACT}

This article examines the role of a translation theory course in a professional training programme, based on a particular pedagogical experience in the Canadian context. The proposed course gives priority to the needs of the learner. Theory is envisaged from the social, rather than the traditional text-based perspective. Course contents and pedagogy are analysed and evaluated.

MOTS-CLÉS/KEYWORDS

approche sociale, enseignement de la traduction, pédagogie, théorie de la traduction

L'enseignement de la théorie de la traduction a encore à trouver sa place au sein de nos programmes de formation professionnelle. Si la formation comprend a priori «la transmission de connaissances théoriques et pratiques» (Valentine 1995: 95), dans les faits, la théorie est perçue le plus souvent comme faisant partie des compétences complémentaires, c'est-à-dire des "matières considérées comme périphériques mais qui viennent renforcer la pratique traductionnelle» à la différence des compétences centrales directement liées à l'acte de traduire » (ibid.: 100). Ainsi, certains programmes prévoient-ils un cours de théorie obligatoire, alors que d'autres offrent plutôt un cours facultatif, sans que l'on s'interroge nécessairement sur la façon dont ce cours devrait contribuer à l'ensemble du programme.

Si l'utilité méthodologique des concepts théoriques commence à être reconnue par rapport aux finalités professionnelles de nos programmes, nous sommes encore loin de savoir comment intégrer ces concepts à l'intérieur d'une démarche pédagogique efficace. "L'enseignement de la traduction ne semble pas encore avoir pleinement profité des retombées des recherches théoriques», affirme Jeanne Dancette. «La question pédagogique demeure entière: comment traduire ces schémas et ces modèles en principes clairs et fiables permettant de guider les nombreux choix que doit effectuer le traducteur dans son travail?» (Dancette 1992: 163). Si la situation s'est quelque peu améliorée au cours des dernières années, Jean Delisle n'en constate pas moins, après une étude exhaustive des manuels de traduction, qu'il «ne semble 
pas y avoir de "consensus" très large quant aux notions constituant le vocabulaire fondamental de l'enseignement de la traduction ». Il s'agit encore d' "une terminologie "jeune" et "éclatée" [...] qui cherche ses mots, pour ainsi dire, afin d'appréhender son objet d'étude» (Delisle 1998: 185)1.

Par ailleurs, l'évolution de la théorie de la traduction contribue elle aussi à problématiser son intégration à part entière aux programmes de formation, du moins au niveau du baccalauréat spécialisé. De plus en plus complexes, les derniers modèles linguistiques, sémiologiques et sociolinguistiques de la traduction sont d'un abord difficile pour les étudiants et étudiantes de premier cycle. Les travaux caractéristiques du virage culturel paraissent plus accessibles. Par contre, axés sur des questions systémiques et macrostructurelles, ils n'éclairent pas forcément les choix microtextuels du traducteur ${ }^{2}$ en apprentissage qui, de prime abord, n'en voit pas toujours la pertinence.

On constate, par conséquent, une certaine polarisation des approches. D'un côté, l'utilité de la théorie serait perçue surtout par rapport à la pratique, c'est-à-dire dans sa dimension méthodologique, et on l'intégrerait à la pédagogie des cours de traduction générale et spécialisée. De l'autre, on privilégierait les finalités de recherche (et non pas de pratique) et l'enseignement de la théorie serait restreint aux programmes de deuxième et de troisième cycles.

La traductologie pourrait-elle échapper à cette dichotomie historique entre pratique et théorie qui hante presque toutes les sciences humaines? Nées de mon expérience pédagogique, les réflexions qui suivent représentent justement une tentative de repenser cette dichotomie, en redéfinissant le contenu et les objectifs d'apprentissage du cours de théorie par rapport au cursus professionnel. En 1997, la direction de l'École de traduction de l'Université York (Toronto) m'a confié la tâche de reprendre en main la version anglaise du cours de théorie de la traduction qui fait partie de notre programme de baccalauréat spécialisé en traduction. Il s'agit d'un cours trimestriel obligatoire de troisième année offert aux anglophones du programme de traduction français-anglais ainsi qu'aux anglophones et aux hispanophones inscrits au certificat de traduction anglais-espagnol et espagnol-anglais. L'année suivante, j'ai mis en place le cours équivalent en français pour les étudiants du programme de baccalauréat spécialisé en traduction anglais-français. Depuis, chaque année, une soixantaine d'étudiants suivent l'un ou l'autre de ces deux cours.

\section{Contexte du remaniement du cours}

Les nouvelles réalités de la pratique professionnelle et leur impact sur les apprenants étaient sans aucun doute les facteurs déterminants dans la décision de remanier le cours. Dans le contexte actuel de la mondialisation et de la localisation, nos étudiants sont confrontés à des défis de plus en plus durs à relever. Souvent ils choisissent la traduction plutôt qu'une autre discipline parce qu'ils aiment les langues, s'intéressent aux différences culturelles, ont le goût du voyage. Dans beaucoup de cas, ils ont même une expérience interculturelle assez approfondie et le choix d'une carrière en traduction répond à leur désir d'améliorer la communication entre groupes linguistiques.

Cependant ces motivations et aspirations personnelles, tout à fait légitimes, résistent difficilement à la confrontation avec les dures réalités et du programme de formation et de la pratique actuelle de la profession. Dans les cours de traduction, les tentatives de conceptualisation pédagogique, différentes d'un professeur à l'autre, 
ont souvent l'effet non voulu d'intimider l'apprenant, en soulignant la technicité et donc la complexité de la démarche traductive, sans lui apporter, du moins dans l'immédiat, l'aide concrète dont il a besoin pour réaliser une traduction de qualtié. S'y ajoute l'insistance inévitable sur les lacunes dans ses connaissances générales que l'on compare défavorablement, avec raison ou non, à celles des générations antérieures d'étudiants. En même temps, le discours ambiant sur le marché du travail, par son accent sur la concurrence, l'évolution rapide des nouvelles technologies et le besoin incontournable de perfectionnement continu, rehausse la perception de l'étudiant des exigences de performance qu'il aura à relever. Perception que d'autres réalités professionnelles (prolifération des logiciels de traduction, fragmentation des marchés locaux, disparition des services et cabinets de traduction et donc de l'encadrement sécuritaire qu'ils assuraient au profit du travail à la pige) ne font que confirmer. Paradoxalement, la reconnaissance professionnelle, en contribuant à faire mieux connaître le travail du traducteur, a ajouté encore aux inquiétudes du langagier aspirant, plus conscient de ses futures responsabilités légales et financières et donc plus en mesure de s'en inquiéter.

Des changements dans le contexte social et économique renforcent cette nouvelle donne. Pour faire face à l'augmentation des frais de scolarité, de plus en plus d'étudiants travaillent à temps partiel sinon à temps plein. Par conséquent, ils ont de moins en moins de temps et d'énergie à consacrer à leurs études, ce qui ajoute à leur stress. Parallèlement, dans la mesure où leurs études leur coûtent plus cher, ils sont plus exigeants à l'égard de leur programme. Reflet peut-être de leur angoisse, cette attitude trouve appui également dans un discours social et gouvernemental qui vante les mérites de la privatisation et qui transforme l'étudiant en client par rapport aux services universitaires. Enfin, une analyse des évaluations des cours de théorie offerts antérieurement démontrait bien qu'un cours trop axé sur des modèles abstraits ne pouvait que solliciter la résistance des étudiants en quête surtout de concepts susceptibles de les aider concrètement à mieux réussir dans leurs cours pratiques.

\section{Principes du remaniement et nouveaux objectifs du cours}

Comment alors repenser le cours de théorie? Le premier principe à respecter consistait justement à formuler les objectifs du cours non pas à partir de compétences théoriques et traductives à acquérir, mais en termes des besoins concrets des étudiants comme aspirants traducteurs. En d'autres mots, il s'agissait de mettre l'étudiant au cœur de son propre apprentissage, en adaptant une approche pédagogique centrée non pas sur les textes théoriques à assimiler, mais sur l'expérience et les besoins de l'apprenant. De plus, ce changement de perspective avait l'avantage de permettre à l'étudiant de se centrer lui-même, puissant antidote contre l'angoisse, de se situer dans son propre cheminement professionnel et d'assumer lui-même la responsabilité de son apprentissage.

Le deuxième principe concernait la façon de concevoir la théorie. Sur ce plan aussi, il fallait changer de stratégie, en abandonnant une perspective textuelle au profit d'une approche sociale plus engagée, pour mettre le traducteur, en tant qu'agent social, au centre de la réflexion théorique. Plus large mais mieux ciblée, cette conception de la théorie permettait à la fois d'interpeller plus directement l'étudiant et de tenir compte davantage de questions professionnelles (statut de la traduction, 
déontologie et éthique professionnelles, contextes de travail, réseautage professionnel, sens d'appartenance à la profession, etc.).

Enfin, il fallait ramener le cours de théorie lui-même de la périphérie au cœur du programme, en l'envisageant non pas comme un ensemble de connaissances complémentaires, mais comme la charpente pédagogique centrale de la formation. En rejoignant les motivations initiales de l'étudiant, en venant nourrir son sens d'appartenance à la profession, en lui donnant un sens de contrôle sur son apprentissage, en reliant la présentation des concepts théoriques au rôle du traducteur comme agent, le cours fournirait en quelque sorte la structure conceptuelle de base à laquelle l'apprenant pourrait ensuite accrocher les différentes connaissances pratiques nécessaires à sa formation.

Conformément à ces changements de perspective, les objectifs du cours ont été reformulés du point de vue de l'apprenant. À la fin du cours, celui-ci doit:

1. avoir une perception meilleure de sa propre identité comme traducteur;

2. mieux connaitre l'histoire et les diverses pratiques actuelles de la profession;

3. mieux apprécier les différentes dimensions du processus de la traduction;

4. mieux comprendre les principales théories linguistiques et interculturelles de la traduction;

5. être mieux en mesure de discuter avec confiance de la traduction avec ses collègues et clients;

6. prendre le recul nécessaire pour analyser sa propre pratique de la traduction.

Dans un sens, les cinq derniers objectifs découlent du premier, primordial, qui touche au cœur de l'identification de l'apprenant avec les exigences et les récompenses de la profession, et lui sert de guide tout au long de son apprentissage.

\section{Contenu du cours}

Pour aider l'apprenant à atteindre ces objectifs, le contenu du cours a été restructuré autour de cinq grandes questions:

1. Qui sont les traducteurs et dans quelles conditions travaillent-ils?

2. Quel est le rôle du traducteur dans la société?

3. Qu'est-ce qui se passe au moment même où l'on traduit?

4. Comment peut-on envisager la traduction du point de vue linguistique?

5. Comment peut-on envisager la traduction du point de vue interculturel?

Ces questions correspondent grosso modo au cheminement de l'apprenant. Dans la première partie du cours, les lectures obligatoires amènent les étudiants à prendre connaissance des précédents et intérêts typiques des traducteurs. Ils découvrent qu'ils partagent bon nombre de ces traits avec les langagiers professionnels, ce qui crée déjà une certaine identification avec la profession. Ils sont exposés en même temps à un assez vaste éventail de pratiques passées et contemporaines de la traduction, et commencent à voir comment ils pourraient trouver leur place dans la profession. Par ailleurs, ils comparent cette façon de voir la traduction «de l'intérieur» comme une activité, avec le point de vue «extérieur» d'un client ou de la société en général qui y voit surtout le texte qui en résulte (Robinson 1997: 6). Nous explorons ainsi les différents stéréotypes de notre société à l'égard de la traduction, nous cons- 
tatons combien celle-ci reste souvent invisible ou dévalorisée, et nous réfléchissons à l'impact de cette réalité sur le statut de la profession et sur notre image de nousmêmes comme professionnels. Sont examinées également les conséquences de cette différence de perspective pour notre façon d'évaluer la qualité d'une traduction (fidélité $v s$ fiabilités) et d'envisager les questions éthiques (valeurs textuelles vs valeurs personnelles et sociales).

La deuxième partie du cours est consacrée au rôle des traducteurs dans la société. Les étudiants prennent conscience de l'importance de la traduction dans l'histoire; ils apprennent que les traducteurs ont inventé des alphabets, contribué à la création des langues et littératures nationales, aidé à diffuser des connaissances et à propager des religions, et fait avancer la science (Delisle et Woodsworth 1995: 23-136, 163189). Pour beaucoup, l'envergure historique de la traduction comme activité et la profondeur de l'engagement social, politique et religieux des traducteurs du passé sont une véritable découverte. Il faut ensuite les amener à voir comment les fonctions du traducteur contemporain sont à la fois sembables et nouvelles par rapport à celles de leurs prédécesseurs. Sur ce plan, 1 'hypothèse de Robinson, quelque peu déconcertante à première vue: "Translation is more about people than words" (Robinson 1997 : 42), les pousse à remettre en question leurs propres préjugés à ce sujet afin d'élaborer une idée personnelle et plus ouverte du rôle social du traducteur contemporain. D'autres lectures sur les traducteurs «comme acteurs sur la scène du pouvoir et le pouvoir» (Delisle et Woodsworth 1995: 137-160) nous amènent à approfondir notre analyse des questions éthiques et à explorer la marge de manœuvre et le pouvoir négotiateur du traducteur contemporain par rapport à ses clients, ses propres valeurs et les exigences de la langue et de la culture d'arrivée.

Dans la troisième section du cours, qui porte sur la traduction comme processus, les étudiants sont encouragés à identifier leur propre façon d'apprendre (privilégientils le visuel, l'auditif ou le kinesthétique, l'analyse ou l'expérience, l'apprentissage en groupe ou le travail indépendant?) et à mieux connaître leurs préférences quant au lieu du travail (ont-ils besoin de tranquilité pour travailler ou sont-ils relativement indifférents à leur environnement?). Ils arrivent ainsi à évaluer de façon plus réaliste le type de travail qui leur conviendrait le mieux. Un exercice d' «introspection à haute voix» ou de "protocole de verbalisation» (Lee-Jahnke 1998: 157) les aide à conscientiser les différentes étapes de leur propre processus traductif, à analyser des moments problématiques, ceux où ils sont les plus aptes à faire des choix malheureux, et à formuler des stratégies concrètes pour maximiser leurs atouts et minimiser leurs faiblesses.

Des lectures pertinentes alimentent cette autoanalyse (Robinson 1997: 93-125). Des discussions en petits groupes donnent aux étudiants l'occasion de se confier leurs inquiétudes et leurs découragements et d'échanger des stratégies pour renforcer leur confiance, créant ainsi un esprit de corps et un sens de solidarité. Axée sur leur vécu comme traducteurs, ne serait-ce que dans le contexte pédagogique, cette partie du cours amène chaque étudiant à mieux se connaître lui-même comme apprenant, alors que les échanges renforcent son sens d'appartenance à la profession. C'est le moment dans le cours où nous soulevons diverses questions reliées à la traduction comme activité: la place de l'intuition dans le processus traductionnel, l'importance du réseautage, les aspirations de carrière, les objectifs et stratégies de perfectionnement à court et à long termes. 
Au moment d'aborder la traduction comme processus linguistique et interculturel, ce qui constitue la partie du cours qui se rapproche sans doute le plus d'un cours de théorie "traditionnel», les étudiants sont donc mieux en mesure de relier un certain éventail de concepts plus abstraits à leur propre pratique, ce qui en facilite l'assimilation. Plusieurs modèles sont présentés. L'objet n'est pas d'amener les étudiants à connaître à fond chacune de ces théories, mais plutôt de leur donner une idée générale de la pensée linguistique sur la traduction. Ici encore, ils sont encouragés à remettre en question leur conception préalable de certaines notions-clés (sens, contexte, équivalence, fonction, etc.), à préciser la compréhension qu'ils en ont d'après leurs cours pratiques et à en explorer l'utilité et l'efficacité opératoire pour leur propre pratique. Sur le plan interculturel, la priorité est accordée à des questions qui sont particulièrement pertinentes pour le contexte canadien: traduction et colonialisme, culture minoritaire vs culture majoritaire, rapports de force entre cultures et langues, traduction et identité culturelle, pratiques féministes de traduction. Les étudiants se rendent compte de l'importance des enjeux institutionnels pour la pratique de la profession et voient comment le contexte social, politique et linguistique influe sur les attitudes et les attentes à l'égard de la traduction comme activité (processus) et comme texte (produit). De nouvelles questions éthiques font surface alors que chaque étudiant approfondit ses propres aspirations à l'égard de son rôle comme agent social.

\section{Format et pédagogie}

Le cours comprend treize séances hebdomadaires de trois heures chacune. Les étudiants ont une liste de lectures obligatoires pour chaque séance. Le principal devoir est un journal de bord (onze ou douze entrées d'une page) où les étudiants notent leurs réflexions sur leurs lectures, leur expérience de la traduction et tout ce qu'ils constatent autour d'eux qui touche à la façon dont on perçoit la traduction dans notre société. La dernière entrée doit comporter une réflexion générale sur leur cheminement au cours du trimestre. Ils doivent aussi faire un compte rendu critique d'un texte théorique ou dresser le portrait d'un traducteur historique ou contemporain. $\mathrm{Si}$, dans le cadre du portrait, une entrevue est possible, c'est une belle occasion pour eux d'échanger avec un traducteur en exercice autour des grands thèmes du cours, de nourrir leur solidarité professionnelle et de commencer le réseautage. Il y a deux tests à livres ouverts. Les questions exigent une réflexion nuancée et structurée sur les grandes questions du cours et visent à vérifier le cheminement de l'étudiant.

L'approche pédagogique privilégie l'interactivité. Les rencontres commencent souvent par un petit exercice d'écriture individuel. Les étudiants sont invités à noter très librement leurs idées sur les questions reliées au thème du jour (exemples: Pourquoi voulez-vous devenir traducteur? Quelles sont pour vous les caractéristiques d'une bonne traduction? Que faites-vous quand vous traduisez? En repensant à votre enfance, quelles sont vos premières expériences de la différence linguistique?). Ces exercices ont pour fonction de ramener l'étudiant à son propre vécu, lui donnant ainsi un ancrage personnel pour la discussion qui suivra. Ils servent en même temps à aiguiser son sens critique, en lui permettant de comparer ses croyances initiales et ses idées reçues, notées spontanément, aux nouvelles données plus nuancées qui ressortent des lectures et des discussions en classe. Je fais souvent un bref tour de table pour solliciter les réactions aux lectures, avant d'en présenter rapidement les points 
principaux en prenant soin de les relier aux grands thèmes du cours. Il y a toujours une période de travail en petits groupes, chaque groupe devant par la suite rapporter en séance plénière les fruits de ses discussions. Dans le cas du cours pour anglophones et hispanophones, il s'est avéré fructueux de demander à un groupe de faire sa présentation en espagnol et d'inviter un étudiant à servir d'interprète simultané vers l'anglais. Ce type d'expérience met les étudiants en situation. Dans la discussion qui s'ensuit, ils parviennent mieux à expliquer les diverses réactions à l'égard de l'interprétation selon que l'on la vit de l'extérieur ou de l'intérieur, à saisir les différences entre la traduction et l'interprétation comme pratique interlinguistique et à évaluer l'impact que peut avoir l'interprétation sur la communication dans un contexte donné.

\section{Évaluation du cours}

Depuis cinq ans, environ 300 étudiants ont suivi le cours en anglais ou en français. Dans l'ensemble, la plupart des étudiants trouvent le cours intéressant, pertinent et stimulant. Les commentaires dans les journaux de bord sont invariablement positifs. Les principaux objectifs du cours semblent en grande partie atteints. Au début du cours, un bon pourcentage d'étudiants expriment des doutes quant à leur choix de carrière, doutes qui se dissipent à mesure qu'ils arrivent à mieux se situer par rapport à la profession. Les lectures historiques servent à revaloriser la traduction et les traducteurs; les étudiants y gagnent en fierté et en sens d'appartenance professionnelle. Les séances consacrées aux modes d'apprentissage et au processus de traduction sont jugés particulièrement efficaces. Les étudiants notent souvent dans leur journal combien cette partie du cours leur a permis de mieux se connaître en tant qu'apprenants. Ils constatent un regain de confiance en eux-mêmes, adoptent de nouvelles stratégies d'apprentissage et en voient des retombées positives dans leurs cours de traduction générale et spécialisée. Ils sont plus à l'aise avec leur choix de carrière et les besoins de perfectionnement qu'elle impose. L'idée du traducteur comme agent social, concept nouveau pour beaucoup d'étudiants, est motivante et valorisante. En laissant une place à l'humain, elle atténue certaines appréhensions à l'égard des nouvelles technologies, offre un cadre de réflexion quant aux questions d'éthique et renforce le sens de la responsabilité professionnelle.

Cela dit, plusieurs améliorations s'imposent pour cibler encore davantage les objectifs. La présentation des différentes pratiques actuelles de la profession devrait être étoffée pour inclure une présentation mieux structurée du marché (entendu ici dans son sens le plus large). Cela pourrait comporter une analyse de la place de la traduction dans les échanges interculturels (la traduction se présente souvent comme une composante d'un autre type de travail), du rôle des institutions gouvernementales (exigences de traduction, mesures d'aménagement linguistique) et des grandes tendances commerciales. On pourrait prévoir de façon plus systématique des activités de réseautage, y compris avec des étudiants d'autres pays, en faisant un meilleur usage d'Internet. Dans le domaine de l'éthique, les étudiants étant à l'affût de renseignements professionnels d'ordre pratique, il serait utile de présenter en plus grand détail le cadre juridique et institutionnel dans lequel le traducteur exerce sa profession. Il reste à décider à quel moment il faudrait introduire ces notions, et de la meilleure façon de les arrimer à l'ensemble du contenu didactique du cours. 


\section{Conclusion}

Les réflexions qui précèdent reflètent évidemment une expérience particulière et, à ce titre, n'ont pas la prétention de s'ériger en préceptes immuables. Si d'anciens étudiants m'écrivent pour me dire combien les concepts abordés dans le cours les aident dans leur travail, seule une analyse plus rigoureuse (sondages auprès d'étudiants, de professeurs et d'employeurs) pourrait valider la pertinence du cours et confirmer son efficacité. Par ailleurs, l'introduction d'un cours de théorie de ce type ayant nécessairement des incidences sur les autres cours du programme, il faudrait évaluer ces incidences de façon plus précise. Des ajustements sont sans doute à faire de part et d'autre pour mieux garantir la cohérence, et donc l'efficacité, du programme de formation dans son ensemble (Voir Whitfield 2002: 118-120).

Sur un plan plus général, cette expérience pédagogique rejoint celle d'autres traductologues contemporains pour faire ressortir l'intérêt de certains principes-clés. Le premier consiste, comme le souligne Mark Shuttleworth, à mieux «cerner exactement ce que nous cherchons à accomplir par l'incorporation d'un élément théorique dans un programme de formation pour traducteurs» (2001: 497). S'il opte pour un cours de théorie axé plutôt sur des questions textuelles et méthodologiques, selon le titre proposé, «Methods and Approaches» (2001: 500), à la différence de l'orientation plus professionnelle que je propose, l'obligation de bien formuler les objectifs visés par rapport à l'ensemble du programme et de la formation n'en demeure pas moins la même. En proposant d'établir ces objectifs en fonction des besoins de ses étudiants, Shuttleworth touche aussi, du moins implicitement, à un deuxième principe qui me semble primordial et que Douglas Robinson énonce avec vigueur dans Becoming a Translator, à savoir l'importance de mettre l'étudiant au centre de notre conceptualisation pédagogique des cours. Faisant appel aux théories connues d'apprentissage, Robinson explore un grand éventail de modes d'apprentissage différents dont il tente de tenir compte au niveau de la conception des exercices et des activités pédagogiques.

Enfin, cette réflexion sur le rôle d'un cours de théorie au sein d'un programme de formation professionnelle s'ouvre forcément sur une interrogation des frontières de ce que nous envisageons sous la rubrique du «théorique». De nouveau, cela recoupe le virage méthodologique ou methodological shift préconisé par Robinson lorsqu'il fait la distinction entre "theory» and «theorizing», entre l'enseignement de «theory and specifically the static form and content of specific theories» (1997: 181) et la transmission pédagogique d'une capacité de théorisation qui permettrait à l'étudiant non seulement d'assimiler des théories reconnues, mais de les adapter et, le cas échéant, d'en formuler d'autres dans le contexte de sa propre activité traductive. Le rôle de l'étudiant en tant qu'agent s'en trouve ainsi renforcé, agent d'abord de son propre apprentissage et de sa propre réflexion théorisante, et responsable ensuite dans sa propre pratique professionnelle et de son rôle d'agent social au sens large du terme.

Une dernière observation s'impose qui touche à une autre dimension du contexte particulier du cours, à savoir son caractère interculturel. Réfléchir sur la théorie de la traduction en deux langues auprès de trois groupes linguistiques distincts réunissant chacun des hommes et des femmes provenant d'une trentaine de pays et d'une assez grande variété de cultures différentes est une expérience pédagogique extrêmement enrichissante. Au fil des discussions en classe, des notions que nous 
abordions comme objets d'étude débordaient souvent leur cadre objectif, se matérialisaient, prenaient des formes parfois insolites, pour venir habiter littéralement l'espace pédagogique, les échanges qui s'y effectuaient et la renégociation explicite et implicite des savoirs qui en découlait. Si l'idée selon laquelle les théories de la traduction reflètent toujours, d'une façon ou d'une autre, les circonstances dans lesquelles elles se sont élaborées a fait son chemin, je crois qu'on est encore loin d'apprécier pleinement l'impact du contexte culturel (ou interculturel) sur l'apprentissage et d'en tenir compte au niveau de la structuration des contenus et des approches pédagogiques.

\section{NOTES}

1. Voir aussim à ce sujet, Cormier, Delisle et Lee-Jahnke 1999.

2. L'emploi du masculin dans cet article n'exclut pas le féminin et se fait dans le seul but d'alléger le texte.

3. Voir Robinson 1997; 6-21.

\section{RÉFÉRENCES}

Dancette, J. (1992) : «L'enseignement de la traduction: peut-on dépasser l'empirisme?», TTR, 5-1, p. 163-179.

Delisle, J. et H. Lee-JAhnKe (dir.) (1998): Enseignement de la traduction et traduction dans l'enseignement, Ottawa, Presses de l'Université d'Ottawa.

Delisle, J., H. Lee-Jahnke et M. C. Cormier (dir.) (1999): Terminologie de la traduction, Amsterdam/Philadelphia, John Benjamins.

Delisle, J. et J. Woodsworth (dir.) (1995): Les traducteurs dans l'histoire, Ottawa, Presses de l'Université d'Ottawa.

Robinson, D. (1997): Becoming a Translator. An Accelerated Course, London/New York, Routledge.

Shuttleworth, M. (2001): «The Role of Theory in Translator Training: Some Observations about Syllabus Design », Meta, 46-3, p. 97-506.

Valentine, E. (1995) : «Réflexions sur les programmes de traduction au Canada», M.-C. Aubin (dir.) Perspectives d'avenir en traduction/Future Trends in Translation, Winnipeg, Presses universitaires de Saint-Boniface: 93-108.

Whitfield, A. (2002): "Teaching Translation Theory: A Canadian Case Study», B. Maia, J. Haller and M. Ulrych (ed.), Training the Language Services Provider for the New Millenium, Porto, University of Porto, p. 111-124. 